\title{
On the Accuracy of Passive Multipath-Aided Underwater Range Estimation
}

\author{
Elizaveta Dubrovinskaya ${ }^{\sharp \star}$, Ivor Nissen ${ }^{\ddagger}$ and Paolo Casari ${ }^{\sharp}$ \\ ${ }^{\sharp}$ IMDEA Networks Institute, Madrid, Spain $\quad{ }^{\star}$ Universidad Carlos III, Madrid, Spain \\ ${ }^{\ddagger}$ Research Department for Underwater Acoustics and Marine Geophysics (FWG), WTD71 Kiel, Germany

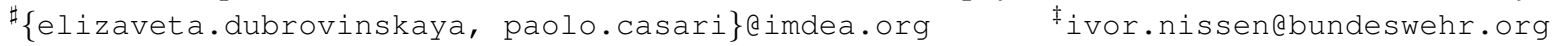

\begin{abstract}
We consider a range estimation algorithm based on the time-of-arrival differences between multipath replicas of a signal transmitted by an anchor node of known location to a silent node that needs to localize itself. We consider the geometry of the problem in the simplified case of a constant sound speed profile (SSP), and argue that the more realistic case of a nonconstant SSP can be still tackled as if the SSP were constant, provided that an "effective" sound speed value is computed based on the geometry of the signal propagation.

We finally evaluate the accuracy of the latter approach in the presence of errors affecting different quantities required for the range estimation process, including the TDoA values and the bottom depth. Our results show that our approach offers improved accuracy with respect to a baseline LSE estimator.
\end{abstract}

Index Terms-Underwater networks, passive localization, autonomous underwater vehicles; multipath; simulation; accuracy

\section{INTRODUCTION AND RELATED WORK}

Extensive ocean investigation is mandated by several applications, e.g., in the conservation, ecology, resource prospection and tactical domains. Autonomous underwater vehicles (AUVs) have the potential to be a key tool for this purpose [1]. However, AUV self-localization remains a challenge due to the characteristics of the underwater environment and to the computational and energy conservation constraints imposed by the AUVs themselves. A variety of different algorithms and architectures to overcome these challenges has been proposed over time (e.g. [1], [2]). Still, several issues remain to be solved. A precise and robust range estimation process, which possibly does not consume an excessive amount of computational resources on AUVs is one of the essential steps in many localization algorithms and advanced inertial navigation systems [3]. Conventional two-way range estimation requires an acoustic transmitter to be integrated on the AUV, which may sometimes be impossible due to size or energy consumption constraints. Without a transmitter available on the node to be localized, range estimation can be performed passively using one-way algorithms, which often require a challenging or timeconsuming synchronization step [4].

A common technique for underwater localization is based on the computation of time differences of arrival (TDoA) among the signals emitted by a set of anchor nodes [5]. Anchor nodes send pings or structured signals, whose TDoA can be measured at the mobile node. The strong aspect of this solution is that it does not require time synchronization. However, an 978-1-5090-2696-8/16/\$31.00 (C)2016 IEEE accurate translation of time differences into distances requires a detailed environmental model, especially in terms of sound speed profile (SSP) and capability to trace the history of signals heard at the receiver. Employing only some average sound speed value might lead to large ranging errors, and may yield results that are valid only as a first-order approximation.

Additional information on the environment can be factored in by analyzing multipath sound propagation. For example, this concept has been applied to the localization of vocalizing mammals in [6], where the authors process the sound recorded by widely spaced receivers, under the assumption that the SSP and the bathymetry of the area are fully known. However, obtaining measurements of the SSP via, e.g., conductivity/temperature/depth (CTD) equipment may be often impractical or exceedingly expensive. The idea of using additional information about multipath propagation for passive range estimation has been proposed in the past (e.g., [7]), and multipath-aided range estimation has been applied to motion tracking for several types of targets [6], [8], [9].

In this paper, we discuss the performance of passive, multipath-aided range estimation between an anchor node and a second node whose location is unknown. We argue that obtaining full knowledge of the environment is often infeasible, and discuss a method to improve the accuracy of ranging by taking into account at least the effect of refraction on sound propagation. The primary advantage of our method is that it leverages on the processing of multipath arrivals at the receiver, and therefore it does not require additional equipment or CTD sensors. However, for the same reason, our approach works preferably in scenarios offering a rich multipath, e.g., shallow waters.

In the remainder of this paper, we introduce the geometry of the problem and the basics of the ranging algorithm (§II), we analyze its accuracy in the presence of different uncertainties (§III) and finally draw concluding remarks in $\S \mathrm{IV}$.

\section{RANGE ESTIMATION ALGORITHM}

\section{A. Scenario and assumptions}

The TDoA-based algorithm considered in this paper is designed to estimate the range between a single anchor node of known position (e.g., a floating GPS [10] or bottom-mounted buoy) and a second node within the coverage range of the buoy's signal. We recall that the latter node is assumed to be passive: therefore, it can only receive the signal from the 
anchor node, but cannot transmit to it. For brevity, in the following we will refer to the anchor node as the "Tx" and to the passive node as the "Rx". We assume that the signal transmitted by the Tx undergoes multipath propagation, so that multiple arrivals of the same signal are detected and distinguished by the Rx. We also assume that the Rx knows the bottom depth and its own depth. The latter can be estimated, e.g., via a pressure sensor, but we note that in the presence of a sufficiently rich multipath it would be possible to partially compensate for this by solving for the depth of the Rx along with its range from the Tx. We also assume that the surface and bottom are flat, which is a common assumption in several shallow water scenario. In any event, the algorithm can be easily extended to accommodate for, e.g., sea bottom slopes.

At the moment a TDoA measurement is taken, the $\mathrm{Rx}$ is considered to be static or moving at a speed that is sufficiently small as to not affect the measurement. We will now proceed by outlining the range estimation algorithm in a homogeneous (i.e., non-refractive) environment, where the SSP is constant and sound wave propagation can be assumed to take place along rectilinear trajectories. We will then extend the algorithm to the more general case of a non-constant SSP.

\section{B. Constant SSP}

In case the SSP can be assumed to be constant throughout the water column, the geometry of the problem is amenable to an analytical solution. Call $d$ the horizontal distance between the Tx and the Rx, and let the notation $\tau_{i j}$ indicate the TDoA between two paths having incurred $i$ and $j$ reflections off the sea surface or bottom. For clarity, we indicate where the reflections have occurred by appending the indices $s$ and $b$ for a surface and a bottom reflection, respectively. For example, $\tau_{02 s b}$ indicates the TDoA between the line-of-sight (LOS) arrival and a second arrival which was reflected twice, first on the surface and then on the bottom. With reference to Fig. 1, we have the following equations:

$$
\begin{array}{lc}
d^{2}= & R_{0}^{2}-\left(h_{R x}-h_{T x}\right)^{2} \\
d^{2}= & \left(R_{0}+c \tau_{01 s}\right)^{2}-\left(h_{R x}+h_{T x}\right)^{2} \\
d^{2}= & \left(R_{0}+c \tau_{01 b}\right)^{2}-\left(2 h_{B}-h_{R x}-h_{T x}\right)^{2} \\
d^{2}= & \left(R_{0}+c \tau_{02 s b}\right)^{2}-\left(2 h_{B}+h_{R x}-h_{T x}\right)^{2} \\
d^{2}= & \left(R_{0}+c \tau_{02 b s}\right)^{2}-\left(2 h_{B}-h_{R x}+h_{T x}\right)^{2}
\end{array}
$$

where $R_{0}$ is the length of the LOS arrival path, $c$ is the constant sound speed value and $h_{R x}, h_{T x}$ and $h_{B}$ are the depths of the Rx, of the Tx and of the bottom, respectively. The solution to the range estimation problem is then found by solving the system of equations above for $d$. We remark that Eqs. (1)-(5) require knowledge of the reflection history of the arrivals. This task is simplified by assuming that the anchor node is deployed either on the surface or on the bottom, so that reflected paths incur, respectively, at least one bottom or one surface reflection. We remark that this is not a restrictive assumption, as anchor nodes for localization systems are likely to be deployed on the surface (to take advantage of GPS fixes) or on the sea bottom at a fixed location (e.g., to avoid being

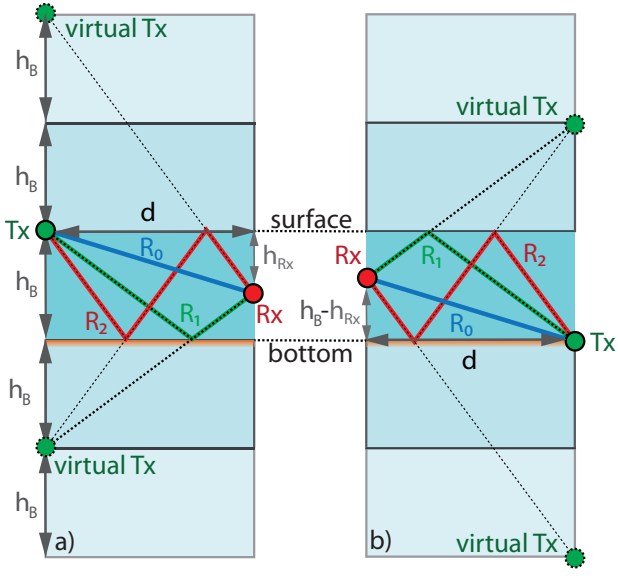

Figure 1. Constant SSP scenario: a) anchor node located on the sea surface; b) anchor node located on the bottom.

damaged by passing ships). In the following, we assume that the $\mathrm{Tx}$ is located on the bottom (right panel in Fig. 1).

\section{Non-constant SSP}

In a typical environment, the SSP varies throughout the water column. In these conditions, the Tx's signal is refracted, possibly leading to non-ideal situations where, e.g., reflected arrivals might be detected earlier than the LOS arrival, or where some arrivals may not exist even in the absence of barriers. Range estimation can still be carried out by fixing some approximate value for $c$ and by finding an approximate solution to (1)-(5), e.g., via a least squares estimator (LSE). In this paper we employ the latter as a reference algorithm, by assuming a fixed sound speed value at $1500 \mathrm{~m} / \mathrm{s}$. However, the TDoAs between the LOS and the reflected arrivals can be employed to infer some information about the propagation of sound between from the Tx to the Rx, and thereby improve the accuracy of range estimates.

In order to do so, we define the effective sound speed $c^{\text {eff }}$ as a measure of the deviation between the rectilinear trajectory joining the $\mathrm{Tx}$ and the $\mathrm{Rx}$ and the actual trajectory followed by two rays for which we compute a TDoA. By recalling that $R_{i}$ and $R_{j}$ are the lengths of the trajectories of arrivals $i$ and $j$ under the assumption of rectilinear propagation, we define the effective sound speed as

$$
c_{i j}^{\mathrm{eff}}=\left(R_{i}-R_{j}\right) / \tau_{i j} .
$$

This value can then be used along with the TDoA between the corresponding pair of arrivals, $\tau_{i j}$, to compute $d$ from Eqs. (1)-(5). As we will show in $\S$ III, the use of $c^{\text {eff }}$ can noticeably improve the range estimation in a number of nonhomogeneous environments. The main drawback is that $c^{\text {eff }}$ must be computed at a moment when the range between the Tx and the Rx is known. However, this is a feasible assumption in many cases. For example, if a ship is deploying a bottom node, the measurement can be taken just prior to releasing the node. Similarly, an AUV could start from a known location, take this measurement, and then begin its mission. 

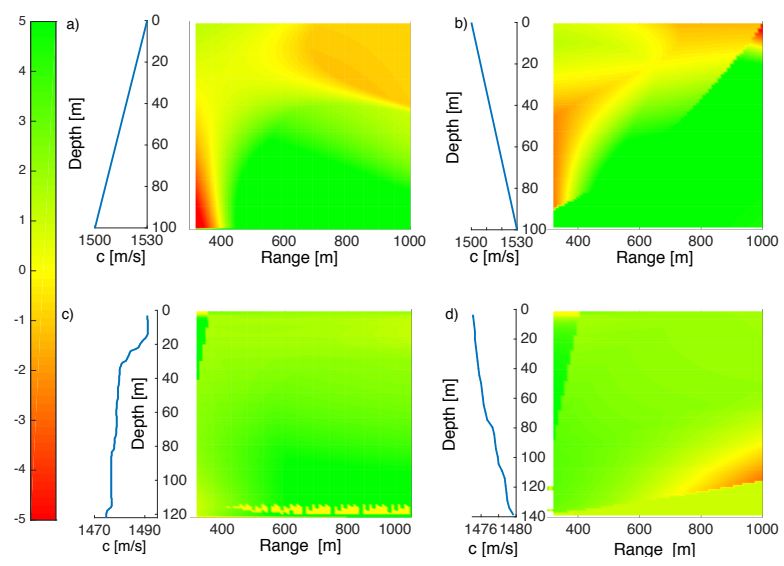

Figure 2. Difference (\%) between the relative error of the LSE range estimator with and without the use of $c^{\text {eff }}$ for different SSPs: a) synthetic upward refractive SSP; b) synthetic downward refractive SSP; c) real summer SSP; d) real winter SSP. The measurement of $c_{i j}^{\text {eff }}$ for each pair of arrivals $i, j$ was taken once at a range of $600 \mathrm{~m}$ and at a depth equal to $0.5 h_{B}$, and then used for the estimation of all other ranges. Green hues denote better performance of the approach based on $c^{\text {eff }}$.

\section{Simulation RESUlts}

In the following we will carry out our performance analysis by modeling acoustic propagation via the Bellhop ray tracing software [11]. Our results were obtained using both simplified upward/downward refractive SSPs and actual SSPs taken from the World Ocean Database [12], in a scenario with flat bottom and surface, and mixed sand/mud bottom sediments. The output of Bellhop was preprocessed to compute TDoAs, which were then employed as an input to the range estimation algorithm. Among all arrivals, we selected three corresponding to LOS, one reflection and two reflections, respectively. If due to specific propagation conditions, one or more of these arrivals are missing, the algorithm tries to compute an estimate using only the remaining ones, or fails otherwise.

\section{A. Performance improvement using $c^{\text {eff }}$}

We start by discussing the accuracy improvement achieved by employing effective sound speed values when computing a solution to Eqs. (1)-(5). Typical range estimation errors in our simulations were found to be between 2 and $30 \mathrm{~m}$, and to have an increasing trend with increasing actual range. Since we are mainly interested in the improvement achieved through the use of $c^{\text {eff }}$, we will discuss the difference between the relative range estimation error in this case and the relative error obtained by assuming $c=1500 \mathrm{~m} / \mathrm{s}$. By calling $e^{\text {eff }}$ the former and $e^{\text {fix }}$ the latter, the performance improvement (or loss) achieved via $c^{\text {eff }}$ is thus computed as $\left|e^{\text {fix }}\right|-\left|e^{\text {eff }}\right|$.

Fig. 2 depicts the relative error using green to red hues. Green hues denote an improvement of the method employing $c^{\text {eff }}$, whereas red hues denote a lower accuracy. In the vast majority of cases, the use of $c^{\text {eff }}$ makes ranging estimates more accurate. However, in some cases the accuracy is worse than by assuming a predefined sound speed value. In particular, we observe that such factors as the depth and range of the $\mathrm{Rx}$, as well as the SSP, affect the accuracy of the estimate.
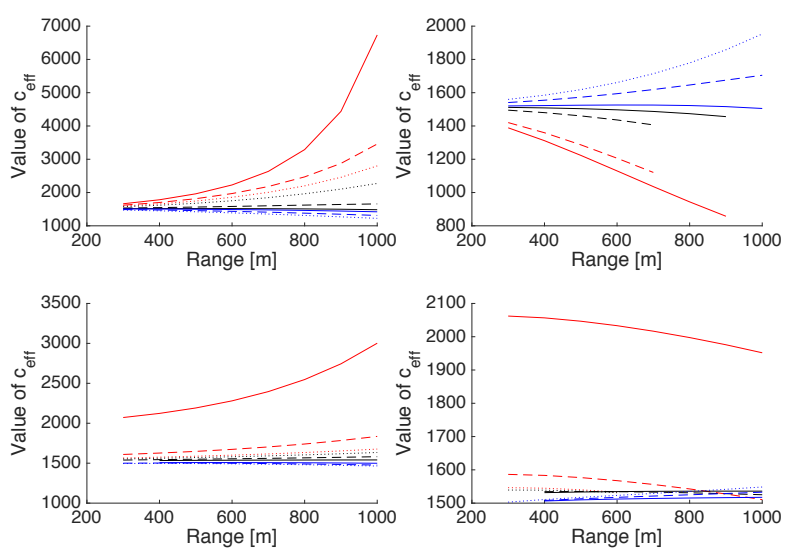

Figure 3. Effective sound speed values $c_{01}^{\text {eff }}$ (red), $c_{02}^{\text {eff }}$ (black) and $c_{12}^{\text {eff }}$ (blue) for the same SSPs considered in Fig. 2, and for different depths of the Rx node (solid: $0.1 h_{B}$; dashed: $0.5 h_{B}$; dotted: $0.9 h_{B}$ ). Notice the different $\mathrm{y}$ axis scale.

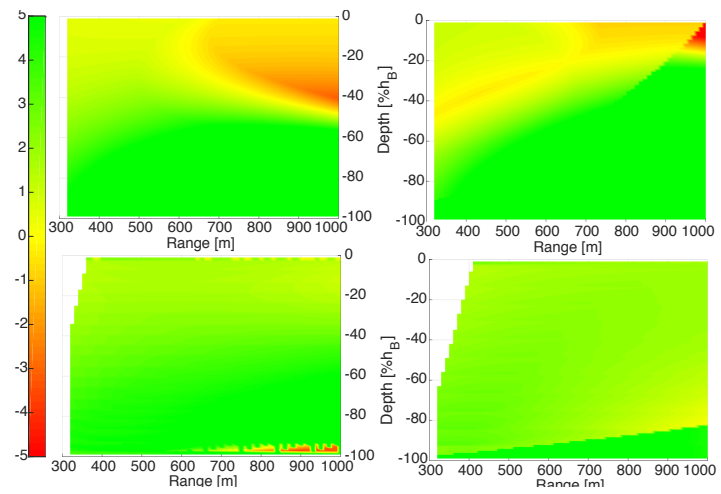

Figure 4. Difference (\%) between the relative error of the LSE range estimator with and without the use of $c^{\text {eff }}$ using the 02-12 arrival pairs, for the same SSPs of Fig.2.

To understand better the reason of this effect, we investigate the variation of $c^{\text {eff }}$ with these three factors. This result is shown in Fig. 3. We observe that $c_{01}^{\text {eff }}$ is the most affected by the range and depth of the Rx, especially when the latter is located close to the surface. We recall that the Tx is located on the sea bottom: hence, this effect is most likely due to the LOS and surface-reflected paths being almost superimposed. Moreover, we observed that in most cases $c_{02}^{\text {eff }}$ and $c_{12}^{\text {eff }}$ tend to evolve symmetrically with respect to some intermediate value that depends on the scenario. This suggests that relying on the TDoA between the corresponding arrival pairs would yield a solution that is less affected by the range and depth of the Rx node. As shown in Fig. 4, this improves the accuracy of the estimation considerably, as relative errors for realistic SSPs are less than $1 \%$ when the node can receive all arrivals (LOS, surface-reflected and surface-bottom reflected) and is not exceedingly close to the surface or bottom.

\section{B. Sensitivity to erroneous input data}

We now focus on the sensitivity of the algorithm to errors affecting the bottom depth, the Tx location the TDoA and the $\mathrm{Rx}$ depth estimates. As our approach is based on TDoA 

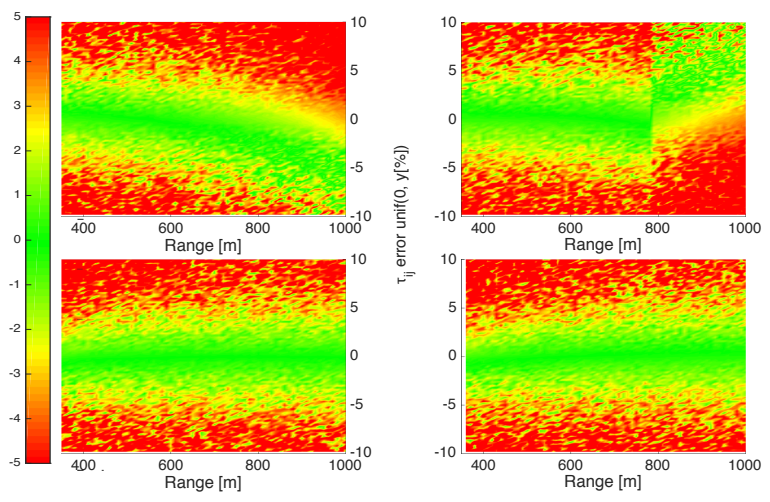

Figure 5. Relative range estimation error $(\%)$ in the presence of errors in TDoA measurements for the SSPs in Fig. 2. The introduced relative error is drawn at random in the interval $[0, y]$, where $y$ is the value reported on each panel's $\mathrm{y}$-axis. The depth of the $\mathrm{Rx}$ is $0.4 h_{B}$.

measurements, a primary requirement is that these measurements are accurate. According to our simulations, quantizing TDoA values down to a precision of less than $10 \mu$ s introduces significant errors. The sensitivity of range estimation to errors on TDoA estimates is shown in Fig. 5. For this evaluation, we introduce on each TDoA value a relative error drawn uniformly at random in the interval $[0, y]$, where $y$ is a tunable parameter. We observe that a setting of up to $y= \pm 5 \%$ yields a relative error of about $5 \%$ on range estimates.

We now assume that the bottom depth $h_{B}$ is affected by a random relative error of up to $\pm 10 \%$ the actual value of $h_{B}$. The impact of this error on the accuracy of the range estimation process depends strongly on the position of the Rx. To support this statement, we show in Fig. 6 the relationship between the error affecting $h_{B}$ and the relative range estimation error. Specifically, when the Rx is not close to the surface or bottom, a $1 \%$ error on $h_{B}$ leads to a relative range estimation error of 2 to $3 \%$. Conversely, if the Rx is located close to the surface or bottom, TDoAs tend to decrease, causing the range estimation error to increase considerably. In addition, Fig. 6 conveys how the ranging error varies for different Tx-Rx distances via the thickness of the line.

Finally, we consider the range estimation performance when the value of the Rx depth $h_{R x}$ is erroneous or not available. In this case, it is still possible to jointly estimate the range and $h_{R x}$. Because, however, there exist two unknowns to be estimated, the ranging process can be completed successfully only when at least three arrivals are received by the Rx. Therefore, the error given by the algorithm strongly depends on the SSP and on the position of the Rx. In turn, the sensitivity of the algorithm to all input data increases, and errors are typically higher than in the previous cases, even if all input data is exact and despite the use of $c^{\text {eff }}$. Fig. 7 shows the relative range estimation error for the SSPs considered so far (see Fig. 2). We observe that due to the absence of a sufficient number of arrivals, in several cases it is not possible to estimate the range. These occurrences are shown as white areas in Fig. 7. For realistic sound speed profiles (bottom panels), the typical relative error was about $3 \%$, and increased
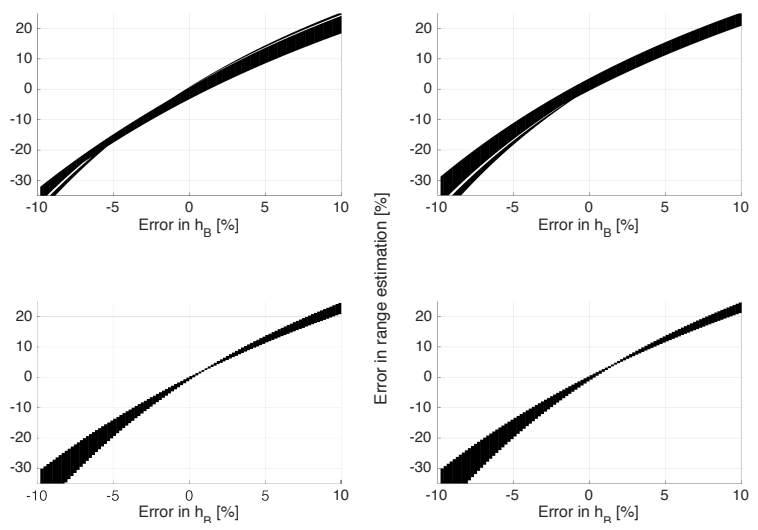

Figure 6. Relative range estimation error $(\%)$ as a function of relative error affecting $h_{B}$, for the same SSPs of Fig. 2. The depth of the Rx is $0.4 h_{B}$. Thicker lines represent a larger variation of the error with the range between the Tx and the Rx for the same error on $h_{B}$.
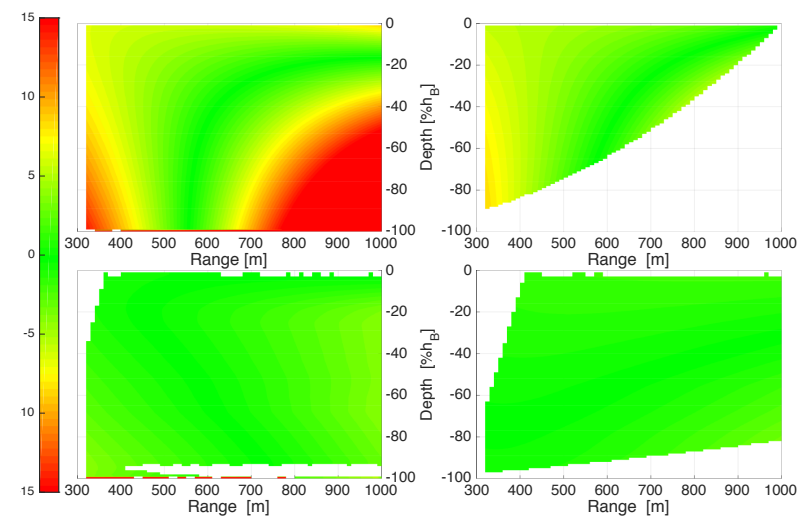

Figure 7. Relative range estimation error [\%] in case the range and $h_{R x}$ are jointly estimated, for the same SSPs reported in Fig. 2.

up to $15 \%$ for the downward refractive profile (top left pane). In any event, the computation of $c^{\text {eff }}$ helped also in this case, as it improved the results by $3-5 \%$.

\section{CONClusions AND FutURE WORK}

In this paper we studied the accuracy of multipath-aided range estimation based on time differences of arrival (TDoA) in the presence of errors on different parameters required to compute the range estimate. We showed that summarizing the propagation history of arrivals via the computation of an effective sound speed value can significantly improve the ranging accuracy. With the proper choice of arrival pairs, the relative error in range estimation in our simulations decreased to values below $1 \%$ for a number of realistic sound speed profiles. We evaluated the sensitivity of the algorithm to different uncertainties affecting the TDoAs, the bottom depth, and considered the case where both the range and the depth of the node must be jointly estimated.

Future work includes implementing an arrival identification algorithm to estimate the history of each multipath arrival, and extending the algorithm to account for node mobility. 


\section{REFERENCES}

[1] L. Paull, S. Saeedi, M. Seto, and H. Li, "AUV navigation and localization: A review," IEEE J. Ocean. Eng., vol. 39, no. 1, pp. 131-149, 2014.

[2] L. Karim, Q. H. Mahmoud, N. Nasser, A. Anpalagan, and N. Khan, "Localization in terrestrial and underwater sensor-based $\mathrm{m} 2 \mathrm{~m}$ communication networks: architecture,classification and challenges," International Journal of Communication Systems, pp. n/a-n/a, 2015. [Online]. Available: http://dx.doi.org/10.1002/dac.2997

[3] T. Zhang, L. Chen, and Y. Li, "AUV underwater positioning algorithm based on interactive assistance of SINS and LBL," Sensors, vol. 16, no. 1, p. 42, 2015.

[4] R. Diamant and L. Lampe, "Underwater localization with timesynchronization and propagation speed uncertainties," IEEE Transactions on Mobile Computing, vol. 12, no. 7, pp. 1257-1269, 2013.

[5] H. G. Thomas, "GIB buoys: an interface between space and depths of the oceans," in Autonomous Underwater Vehicles, 1998. AUV'98. Proceedings of the 1998 Workshop on, Aug 1998, pp. 181-184.

[6] C. O. Tiemann, M. B. Porter, and L. N. Frazer, "Localization of marine mammals near Hawaii using an acoustic propagation model,"
The Journal of the Acoustical Society of America, vol. 115, no. 6, pp. 2834-2843, 2004.

[7] B. Friedlander, "Accuracy of source localization using multipath delays," IEEE Transactions on Aerospace and Electronic Systems, vol. 24, no. 4, pp. 346-359, 1988.

[8] P. Blanc-Benon and C. Jauffret, "TMA from bearings and multipath time delays," IEEE Transactions on Aerospace and Electronic Systems, vol. 33, no. 3, pp. 813-824, 1997.

[9] R. Morrissey, J. Ward, N. DiMarzio, S. Jarvis, and D. Moretti, "Passive acoustic detection and localization of sperm whales (physeter macrocephalus) in the tongue of the ocean," Applied Acoustics, vol. 67, no. 11, pp. 1091-1105, 2006

[10] H. Thomas, "GIB buoys: An interface between space and depths of the ocean," Proceedings of IEEE Autonomous Underwater Vehicles, 1998.

[11] M. B. Porter, "The bellhop manual and users guide: Preliminary draft," Heat, Light, and Sound Research, Inc., La Jolla, CA, USA, Tech. Rep, 2011.

[12] "World ocean database." [Online]. Available: https://www.nodc.noaa. gov/OC5/WOD13/ 